\title{
SSinteza
}

Impact of Internet on Business Activities

\section{EMPIRICAL ESTIMATION AND COMPARISON OF NORMAL AND STUDENT-T LINEAR VAR ON THE BELGRADE STOCK EXCHANGE}

\author{
Zoran Jeremić, Ivica Terzić \\ Singidunum University, Belgrade
}

\begin{abstract}
:
In this paper we describe theoretical and empirical linear VaR for the cases where the stock portfolio returns and the risk factor returns follow a normal distribution and Student $t$ distribution. The main aim of this study is to show the effect that leptokurtosis has on VaR estimate. Using the daily Belex 15 data from January 2011 to January 2014 we estimate 1-day Normal and Student $t$ VaR for different significance levels. The results show that for low significance levels, the normality assumption can overestimate VaR if the return distribution is leptokurtic. On the other hand, for higher significance levels, the normality assumption can seriously underestimate VaR. In the case of Serbian stock market the assumption of Student's t-distribution leads to VaR estimates that are more representative of historical behavior of Belex 15 than normal linear VaR.
\end{abstract}

\author{
Key words: \\ Linear VaR, \\ Serbian stock market, \\ normal distribution, \\ Student-t distribution, \\ risk estimation.
}

\section{INTRODUCTION}

Risk has become a growing concern for most financial institutions today and they must have a robust risk management framework in place if they wish to survive in a more interconnected and complex financial world. One of the most important tasks of financial institutions is evaluating and controlling exposure to risk. This task is called financial risk management. Depending on the sources of risk, a financial institution may face market, credit or operational risk. Market risk describes the portfolio exposure to the moves of certain market variables. There are four standard market risk variables: equities, interest rates, exchange rates, and commodities. A financial instrument is dependent on those market factors and its price fluctuates as the underlying market factors move. Market risk arises from variations in equity prices, interest rates, exchange rates, and commodity prices. The exposure to market risk can be measured by changes in the portfolio value, or by profits and losses.

A risk statistic called Value-at-Risk (hereinafter VaR) has arisen as a powerful tool to capture market risk exposure of portfolio, sector, asset class or security over some specified period of time. Regulators and the financial industry advisory committees recommend $\mathrm{VaR}$ as a way of measuring risk. Today, VaR measurement methodology is a widely-used tool in financial market risk management in developed countries.
The main characteristic of VaR is that of synthesizing in a single value the possible losses which could occur with a given probability in a given temporal horizon [1]. VaR has been estimated by a number of methods, including variance-covariance (also called parametric or Analytical), Historical simulation and Monte Carlo simulation methods $[2,3,4]$. The VaR methodologies mainly differ in ways of constructing the probability density function. The focus of our research in this paper will be on analytical approach for determining the VaR. The essence of analytical method is that it assumes a normal distribution.

Market price movements often display patterns that differ from the statistical simplifications used in modeling analytic linear VaR. The returns on most financial assets are skewed and leptokurtic random variables, although the extent of leptokurtosis depends on the sampling frequency and the market in question. A negative skewness is often evident in daily equity index and stock returns because large price falls are often succeeded by further price falls as investors try to close out positions to limit losses. Nevertheless, the standard analysis of financial asset returns most often begins with an assumption that returns are normally distributed, and majority of risk models are based on the normality assumption.

Although there are many studies that calculate VaR using the parametric approach, i.e. normal linear $\mathrm{VaR}$, there are rare research papers that introduce leptokurtosis effect 
into VaR estimation and compare results provided by different distribution assumptions.

A leptokurtic density is one that has a higher peak than a normal density. Since the total area under the curve must be 1, a higher peak implies that the tails of a unimodal density are also heavier. Hence a unimodal leptokurtic density has heavy tails (fat tails), i.e. positive excess kurtosis. [14]. Leptokurtosis is one of the basic stylized facts emerging from examination of the empirical distributions of financial asset returns [12].

Even in developed financial markets, the empirical research on VaR estimation where the stock portfolio returns are generated by Student $t$ distribution is very scarce. The situation is much more complex when we take into account emerging stock markets. Thus, the objective of this paper is to extend return and risk analysis in Serbian financial market on nonnormal assumptions in order to produce VaR estimates and see are they more representative than $\mathrm{VaR}$ figures derived by normal linear VaR. Estimation of linear VaR for the case where returns are generated by both distributions will be empirically applied and tested on Serbian stock market in period from January 2011 to January 2014. Although VaR is conceptually straightforward, when we want to estimate linear $\mathrm{VaR}$ where the stock portfolio returns are generated by Student $t$ distribution, it becomes computationally challenging. Of course, $\mathrm{VaR}$ calculation can be derived by the use of commercially available simulation packages. However, such packages are generally costly and inflexible and $\mathrm{VaR}$ calculations predominantly are based on normal distribution. Thus, this paper is one of the first papers which demonstrates that the calculation of Student $t$ linear VaR can be performed using flexible computational power of Microsoft Excel and Solver add-in.

Contribution of this paper is threefold. Firstly, to the best our knowledge no one so far has investigated relative performance of analytical $\mathrm{VaR}$ approach based on different distribution assumptions and compared the obtained results in the Serbian stock markets during observed risk horizon. Secondly, contribution is to extend the very scarce empirical research on VaR estimation in this financial market and to provide valuable information to regulators and financial institutions for setting market risk capital requirements and evaluating the market risk exposure of their trading portfolios based on VaR methodology. Thirdly, we demonstrate in detail how well the Student-t distribution behaves in estimating VaR measure for stock market index and illustrate the process for determining the degrees of freedom of the Student- $t$ distribution, which is required in VaR estimation.

The rest of the paper is organized as follows: Section 2 briefly summarizes the literature review with similar researches. Section 3 presents descriptions of basic density functions which are incorporated in our VaR calculations, derivation of analytical formula for linear $\mathrm{VaR}$ and methodologies for determining the degrees of freedom of the Student-t distribution. Section 4 gives the description of the analyzed data and statistical characteristics of Serbian stock market. Findings and results are also presented and discussed in section 4 . Section 5 concludes by summarizing the main results of empirical research.

\section{LITERATURE REVIEW}

Far as we know only a few research papers take into account leptokurtosis effect of asset returns to VaR estimation. One of the first applications of the Student $\mathrm{t}$ distribution to VaR estimation was by Huisman et al. They investigated and compared different parametric VaR estimators for be-weekly returns on US stock and bonds and provided evidence that parametric VaR models which incorporate the fat tails measure accurately market risk exposure among other comparable VaR models [5]. Since the normal model for factor distributions is overly simplistic, Fong and Vasicek suggest estimating the probability distribution of the portfolio value changes by the gamma distribution. Thus, they used the approximation for the portfolio VaR value based on a generalized gamma distribution. The gamma distribution takes into consideration the skewness of the distribution, whereas the normal distribution is symmetric and does not reflect the skewness [6]. Monteiro calculated VaR using four alternative distributional forms to assess the hedge funds indexes market risk. One of the distributional forms was the t-student distribution [7]. Recently, Lin and Shen studied the empirical performance of value-at-risk calculations using Student- $t$ distributions. The main finding of their study is that using the student-t distribution for estimating VaR can improve the VaR estimation and offer accurate VaR estimates, particularly when tail index technique is used to determine the degrees of freedom and the confidence level exceeds 98.5 percent [8]. Yoon and Kang investigated two Japanese financial data sources, the Nikkei 225 Index and JPY-USD exchange rate, using the symmetric FIGARCH and asymmetric FIAPARCH models with normal, Student-t, and Skewed Student-t distribution innovations. From the results of analysis, they found that the skewed Student-t FIGARCH and FIAPARCH VaR models for long and short positions predict critical loss more accurately than models with normal and Student- $t$ innovations [9]. Hsu Ku investigated major equity and currency markets in the US, Japan and the UK and found that exist significant second-moment transmission effects and obvious time-varying patterns of correlation coefficients among those markets. On the other hand, he found that the multivariate Student-t distribution is suitable for analyzing the visible leptokurtosis that is common in financial markets. Thus, he conducted comparison on the hedging efficiency of hypothetical portfolios consisting of stock and currency future positions in order to justify the multivariate Student-t distribution based on the DCCMGARCH model [10]. Rozga and Arnerić theoretically presented the dependence between volatility persistence, kurtosis and degrees of freedom from Student's t-distribution in estimation alternative risk measures on simulated returns. The comparison procedure of $\mathrm{VaR}$ estimation they established with assumption that returns follow extreme value distribution, precisely Student's t-distribution with non-integer degrees of freedom [11]. 


\section{METHODOLOGY OF RESEARCH}

\section{Definition of VaR}

$\mathrm{VaR}$ is an attempt to provide a single number that summarizes the total risk in a portfolio [12]. VaR is a loss that we are fairly sure will not be exceeded if the current portfolio is held over some period of time [13]. When using the value at risk measure, we are interested in making a statement of the following form:

"We are (1- $\alpha)$ percent certain that we will not lose more than V dollars in time $h . "$

The variable $\mathrm{V}$ is the $\mathrm{VaR}$ of the portfolio. It is a function of two parameters: the time horizon, $h$, and the confidence level, (1- $\alpha$ ) percent. It is the loss level during a time period of length $h$ that we are (1- $\alpha) \%$ certain will not be exceeded.

\section{Mathematical Definition of linear VaR}

In our study we use parametric (analytic) approach, linear VaR with assumptions that the changes in the portfolio value over the time horizon are normally and nonnormally distributed.

Table 1 shows the mean and variance for two probability distribution functions that have been used in financial modeling and risk management. Our empirical research of estimation of VaR figure is based on these two distributions.

An analytic formula for the $\mathrm{VaR}$ for a portfolio with an i.i.d. normal return is as follows:

Table 1. Distributions and Their Mean and Variance

\begin{tabular}{|c|c|c|c|c|}
\hline & Density function & Parameters & Mean & $\begin{array}{c}\text { Vari- } \\
\text { ance }\end{array}$ \\
\hline Normal & $f(x)=\frac{1}{\sigma \sqrt{2 \pi}} e^{-\frac{1}{2}\left(\frac{x-\mu}{\sigma}\right)^{2}}$ & $\begin{array}{c}\mu \text { location } \\
\sigma \text { scale } \\
\sigma>0\end{array}$ & $\mu$ & $\sigma^{2}$ \\
\hline Student - t & $f(x)=\frac{1}{\sqrt{\pi \cdot n}} \cdot \frac{\Gamma\left(\frac{n+1}{2}\right)}{\Gamma\left(\frac{n}{2}\right)} \cdot\left(1+\frac{x^{2}}{n}\right)^{-\frac{n+1}{2}}$ & $\begin{array}{c}n \text { degrees of } \\
\text { freedom }\end{array}$ & 0 & $\frac{n}{n-2}$ \\
\hline
\end{tabular}

$$
\operatorname{VaR}_{\alpha}=\Phi^{-1}(1-\alpha) \sigma-\mu
$$

where $\Phi^{-1}$ is the standard normal distribution function with expectation $\mu$ and standard deviation $\sigma$.

Parametric linear VaR formula for the case where the portfolio's returns are generated by a Student $t$ distribution is as follows:

$$
\begin{aligned}
& \text { Student } t \operatorname{Var} r_{\alpha, n}= \\
& \sqrt{n^{-1}(n-2)} t_{n}^{-1}(1-\alpha) \sigma-\mu
\end{aligned}
$$

where $t_{n}^{-1}$ denote standard Student $t$ distribution and $n$ is the degrees of freedom, which controls the tail thick- ness of the distribution. Since quantiles translate under monotonic transformations, the a quantile of the standardized Student $t$ distribution with $n$ degrees of freedom, i.e. the Student $t$ distribution with mean 0 and variance 1 , is $\sqrt{n^{-1}(n-2)} t_{n}^{-1}(\alpha)$.[12]

In contrast to the normal distribution, the Student- $t$ distribution is estimated with an additional parameter $n$, which stands for the number of degrees of freedom measuring the degree of fat-tails in the density. The $t$ distribution is closely related to the normal distribution: it is a symmetric curve that converges to the standard normal density as the degrees of freedom increase. The degrees of freedom is the only parameter in the $t$ distribution and the lower the degrees of freedom the lower the peak of the distribution and the longer the tails [1].

The critical value $t_{n}^{-1}(1-\alpha)$ and $\Phi^{-1}$ can be found in statistical tables or using the Excel function TINV and NORMSINV. But before finding critical values for TINV we have to estimate the degrees of freedom parameter. The degrees of freedom parameter $n$ is estimated by fitting the distribution using maximum likelihood estimation (MLE). The MLE process is based on the construction of a likelihood function, which depends on the parametric form that is assumed for the distribution and on the sample data. Then the parameters of the distribution are chosen to maximize this function. These values are the maximum likelihood estimators. Alternatively, a quick approximation to $n$ may be obtained using a simple moment matching method called the method of moments (MM), which entails equating the sample moments to population moments. We shall apply and compare both methods in the empirical research.

We will assume that the conditional mean of the return, $\mu_{t}$, is simply zero in both normal and Student $t$ linear VaR formulas. For daily data this is a quite reasonable assumption. Hence, under the normal and Student $t$ distributional forms, the VaR estimates are equal to the product of the forecast portfolio standard deviations by the appropriate percentile. We will forecast standard deviation using sample hystorical volatility model.

\section{DATA AND EMPIRICAL FINDINGS}

Estimation of linear VaR for the case where returns are generated by both distributions is empirically applied on Serbian stock market in order to illustrate the impact and effect of leptokurtosis on the VaR estimate. We shall assume that $\mathrm{VaR}$ is measured at the portfolio level, without considering the mapping of portfolios to their risk factors. We use the four most commonly used confidence levels, the $90 \%, 95 \%, 99 \%$ and $99,5 \%$ confidence levels. The target horizon is one day, which is equal to the index return series frequency. Typically $\mathrm{T}$ is chosen in practice to be be- 
tween 250 and 1000 days corresponding to approximately 1 to 4 years [15]. The models which we use to compute the $\mathrm{VaR}$ estimates are described in section 3.

This paper considers time series data set of Serbian capital market: index Belex 15. Daily historical data on the Serbian blue chip stock index (Belex 15) from 1 January 2011 until 1 January 2014 are downloaded from Belgrade stock exchange. The daily price series are converted into the daily percentage logarithmic return series. The returns at time $\mathrm{t}$ are calculated by $r_{t}=\ln \left(P_{t} / P_{t-1}\right) * 100$ for $t=$ $1,2, \ldots, \mathrm{T}$, where $\mathrm{P}_{\mathrm{t}}$ is the current price and $\mathrm{P}_{\mathrm{t}-1}$ is the previous day's price.

When using financial models to assess risk management and portfolio allocation decisions, in order for the model assumptions to be understood and rationalized, it is important to appreciate the potential shape and behavior of asset return distributions. The purpose of this section is to firstly analyze the shape and characteristics of the index return distributions during three years period (2011-2014).

Table 2 shows the descriptive statistics of the index daily returns. The average daily return is close to zero. The unconditional standard deviations is $0,96 \%$. Belex 15 exhibits kurtosis above 3, i.e. 14,76. Another feature of the return series is the presence of skewness. In fact, index is slighty negatively skewed. Additional tests of mean and skewness performed at at 5\% significance level concludes that mean and skewness are not significantly different from zero. The quartiles shown in table (Q1, Q3) inscribe $50 \%$ of the values in the sample. The fat-tailed and symmetric properties of return distribution motivate the use of Student- $t$ distribution in this study.

Using the daily Belex 15 data from 1 January 2011 to 1 January 2014 shown in Fig. 1, we estimate the degrees of freedom parameter for a generalized Student $t$ distribution representation of the daily returns, using the MM and MLE, as described in section 3. Then we compute the 1 -day Student $t \mathrm{VaR}$ at the $0.5 \%, 1 \%, 2.5 \%, 5 \%$ and $10 \%$ significance levels, as a percentage of portfolio value, using both estimates for the degrees of freedom parameter. We also compare the Student $t$ linear VaR figures with the normal linear VaR over a 1-day horizon.

In Excel spreadsheet we compute the sample of daily log returns, and then standardizes the observations to have a sample mean of 0 and a sample variance of 1 . Then we assume a value for the degrees of freedom and calculate the log likelihood for the standardized $t$ distribution, based on the density function shown in Table 1 (second row) for each observation. Summing the log likelihood of each observation gives us a value for the log likelihood function based on our assumed value for the degrees of freedom. Finally, we apply the Excel Solver to find the degrees-of-freedom parameter which maximizes our log likelihood function. The sample mean log return is $-0.02 \%$ and the sample standard deviation of log returns is $0.96 \%$. Using these values to normalize the sample, we then construct the log likelihood function as explained above and maximize it using the Solver. The result is a degrees-offreedom estimate of 3.36. The method of moments gives an estimate $n=4.41$ for the degrees of freedom parameter.
The obtained results for both estimates for the degrees of freedom parameter are summarized in Table 3.

Table 1. Descriptive Statistics of the Belex 15 Return Series

\begin{tabular}{|l|l|}
\hline \multicolumn{2}{|l|}{ Descriptive Statistics } \\
\hline AVERAGE: & $-0,02 \%$ \\
\hline STD DEV: & $0,96 \%$ \\
\hline SKEW: & $-0,09$ \\
\hline EXCESS-KURTOSIS: & 14,76 \\
\hline MEDIAN: & $-0,02 \%$ \\
\hline MIN: & $-7,41 \%$ \\
\hline MAX: & $8,23 \%$ \\
\hline Q 1: & $-0,50 \%$ \\
\hline Q 3: & $0,45 \%$ \\
\hline
\end{tabular}

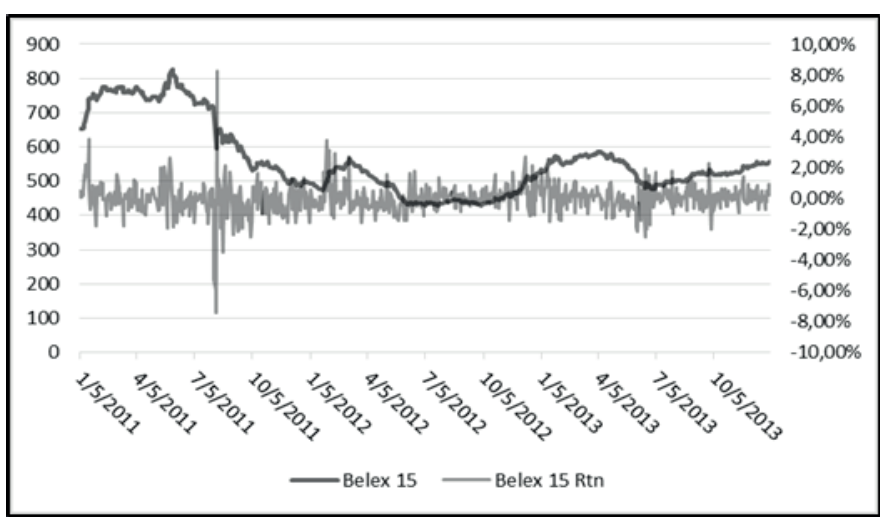

Figure 1. Evolution of Belex 15 daily index prices (on the left) and daily log returns (on the right) for period from 1 Jan 2011 to 1 Jan 2014.

The resulting estimates and comparison of 1-day Student $t \mathrm{VaR}$ at the different significance levels for the method of moments and maximum likelihood estimates and normal linear VaR over a 1-day horizon are displayed in Table 4.

The 1-day Student $t \mathrm{VaR}$ is considerably greater than the normal $\mathrm{VaR}$ at the $0.5 \%$ and $1 \%$ significance levels, it is a little greater than the normal $\mathrm{VaR}$ at the $2.5 \%$ level, and at the $5 \%$ and $10 \%$ significance levels the normal VaR is greater than the Student $t \mathrm{VaR}$. This is because the tails of the Student- $t$ density have greater mass and the peak at the centre is higher than the normal density with the same variance. Hence, for quantiles lying further toward the centre there may be less mass in the tail of the Student $t$ density than in the tail of the normal density.

We can conclude that for low significance levels (e.g. $5 \%$ or $10 \%)$, the normalty assumption can overestimate $\mathrm{VaR}$ if the return distribution is leptokurtic. For higher significance levels (e.g. $0.5 \%-2.5 \%$ ), the normality assumption can seriously underestimate $\mathrm{VaR}$ if the return distribution is leptokurtic.

The results show that the model risk arising from the assumption that returns are normally distributed is very significant, especially when $\mathrm{VaR}$ is measured at high confidence levels such as $99.5 \%$ and $99 \%$. The Student $t \mathrm{VaR}$ 
model provides a more accurate representation of Serbian financial asset returns, but a potentially significant source of model risk arises from assuming the return distribution is symmetric.

Table 3. Different Estimates for the Degrees of Freedom parameter of Student-t distribution

\begin{tabular}{|l|c|}
\hline \multicolumn{2}{|l|}{ Calibration of Student $\mathbf{t}$ - MLE } \\
\hline Degrees of Freedom & 3,36 \\
\hline Mean & $-0,02 \%$ \\
\hline Standard Deviation & $0,96 \%$ \\
\hline Method of Moments \\
\hline XS Kurtosis \\
\hline Population XS Kurtosis & 14,76 \\
\hline Parameter Fitting & 14,76 \\
\hline Degrees of Freedom & MM \\
\hline Objective & 4,41 \\
\hline
\end{tabular}

Table 4. Estimated Student $\mathrm{t}$ and Normal VaR

\begin{tabular}{|c|c|c|c|c|c|}
\hline \multicolumn{7}{|c|}{ VaR Parameters } \\
\hline $\begin{array}{c}\text { Significance } \\
\text { Level }\end{array}$ & $0,5 \%$ & $1 \%$ & $2,5 \%$ & $5 \%$ & $10 \%$ \\
\hline $\begin{array}{c}\text { Risk Hori- } \\
\text { zon (days) }\end{array}$ & 1 & 1 & 1 & 1 & 1 \\
\hline \multicolumn{5}{|c|}{ VaR } \\
\hline t-VaR (MLE) & $3,60 \%$ & $2,81 \%$ & $1,97 \%$ & $1,46 \%$ & $1,02 \%$ \\
\hline t-VaR (MM) & $3,30 \%$ & $2,69 \%$ & $2,00 \%$ & $1,54 \%$ & $1,11 \%$ \\
\hline Normal VaR & $2,48 \%$ & $2,24 \%$ & $1,89 \%$ & $1,59 \%$ & $1,24 \%$ \\
\hline
\end{tabular}

\section{CONCLUSION}

Ongoing global financial crisis of 2007/2008 has showen that our current understending of risk is not sufficient in order to measure risk properly, even in developed countries, and hance prevent black swans in the financial markets. Traditional models in finance rely heavily on the use of the normal (Gaussian) distribution. VaR measures are often calculated under the assumption that the underlying return series are normally distributed. There is ongoing discussion about the return distribution and risk in financial markets. Academics try to model these distributions in order to gain a better undrestending how returns and risk are distributed and can be measured.

In this paper we showed the effect that leptokurtosis has on $\mathrm{VaR}$ estimate and indroduced better measures of risk from current existing mesures in Serbian financial institutions. Using the daily Belex 15 data from January 2011 to January 2014 we estimated 1-day Normal and Student $t$ linear VaR for different significance levels. The results show that for low significance levels, the normality assumption can overestimate $\mathrm{VaR}$ if the return distribution is leptokurtic. On the other hand, for higher significance levels, the normality assumption can seriously underestimate VaR. In the case of Serbian stock market the Student $t$ distribution produces $\mathrm{VaR}$ estimates that are more representative of historical behavior of Belex 15 than normal linear VaR.

We conclude that VaR based on normal distribution assumption is not suitable for financial risk measurement in Serbian stock market, and provide new evidence that more complex and complicated extensions of VaR models based on fat tailed distribution are well suited for this market. These results might be valuable for investors and financial institutions in Serbia in order to improve internally developed risk models.

In order to additionally confirm the findings in this study, it is necessary to model volatility as a key input into the VaR calculation and implement a backtests, which will be the subject of our further researches.

\section{REFERENCES}

[1] S. Rachev, C. Menn and F. Fabozzi, Fat-Tailed and Skewed Asset Return Distributions. New Jersey: John Wiley \& Sons, Inc., 2005.

[2] D. Hendricks, "Evaluation of value-at-risk models using historical data”, Federal Reserve Bank of New York Economic Policy Review, 1996, pp. 39-69

[3] T. Beder, "VAR: Seductive but dangerous", Financial Analysts Journal, 1995, pp.12-24

[4] J. Mahoney, "Forecast biases in value-at-risk estimations: Evidence from foreign exchange and global equity portfolios”. LSE Financial Markets, Special Paper Series, no. 93., 1997

[5] R. Huisman, G. Koedijk and A. Pownall, "VaR-x: Fat tails in financial risk management", Journal of Risk, 1998, pp. 47-61

[6] G. Fong and O. Vasicek, "A Multidimensional Framework for Risk Analysis”, Financial Analyst Journal, 1997, pp. 5158

[7] P. Vitor Monteiro, "Forecasting Hedge Funds Volatility: A Risk Management Approach”, March 2004. Available at SSRN: http://ssrn.com/abstract=570065

[8] C-H. Lin and S-S. Shen, "Can the student-t distribution provide accurate value at risk?", Journal of Risk Finance, vol. 7, iss: 3, 2006, pp. $292-300$

[9] S-M. Yoon and S-H. Kang, "A Skewed Student-t Valueat-Risk Approach for Long Memory Volatility Processes in Japanese Financial Markets", Journal of International Economic Studies, vol. 11, No. 1, June 2007, pp. 211-242

[10] Y-H. Hsu Ku, "Student-t distribution based VARMGARCH: an application of the DCC model on international portfolio risk management", Applied Economics, vol. 40, Iss. 13, 2008, pp. 1685-1697

[11] A. Rozga and J. Arnerić, "Dependence between volatility persistence, kurtosis and degrees of freedom", Revista Investigacion operacional, vol., 30, No.1, 2009, pp. 32-39

[12] C. Alexander, Value at Risk Models. Chichester: John Wiley \& Sons Ltd., 2008.

[13] J. Hull, Risk Management and Financial Institutions. New Jersey: John Wiley \& Sons, Inc., 2012.

[14] C. Alexander, Market Risk Analysis, Quantitative Methods in Finance, Volume 1, Chichester: John Wiley \& Sons Ltd., 2008.

[15] P. Cristofersen, Elements of financial risk management. Waltham: Academic Press, 2012. 